\title{
Detection of Wolf-Rayet stars in host galaxies of gamma-ray bursts (GRBs): are GRBs produced by runaway massive stars ejected from high stellar density regions? ${ }^{\star}, \star \star$
}

\author{
F. Hammer ${ }^{1}$, H. Flores ${ }^{1}$, D. Schaerer ${ }^{2,3}$, M. Dessauges-Zavadsky ${ }^{2}$, E. Le Floc'h ${ }^{4, * *}$, and M. Puech ${ }^{1}$ \\ 1 Laboratoire Galaxies Étoiles Physique et Instrumentation, Observatoire de Paris-Meudon, 5 place Jules Janssen, \\ 92195 Meudon Cedex, France \\ e-mail: francois.hammer@obspm.fr \\ 2 Observatoire de Genève, 51 Ch. des Maillettes, 1290 Sauverny, Switzerland \\ ${ }^{3}$ Laboratoire d'Astrophysique Toulouse-Tarbes, UMR 5572, 14 Av. E. Belin, 31400 Toulouse Cedex, France \\ ${ }^{4}$ Steward Observatory, University of Arizona, 933 North Cherry Avenue, Tucson, AZ 85721, USA
}

Received 6 January 2006 / Accepted 13 March 2006

\section{ABSTRACT}

\begin{abstract}
We have obtained deep spectroscopic observations of several nearby gamma-ray burst (GRB) host galaxies revealing for the first time the presence of Wolf-Rayet (WR) stars and numerous O stars located in rich and compact clusters or star forming regions. Surprisingly, high spatial resolution imaging shows that the GRBs and the associated supernovae did not occur in these regions, but several hundreds of parsec away. Considering various scenarios for GRB progenitors, we do not find any simple explanation of why they should be preferentially born in regions with low stellar densities. All the examined GRBs and associated SNe have occurred 400 to $800 \mathrm{pc}$ from very high density stellar environments including large numbers of WR stars. Such distances can be travelled through at velocities of $100 \mathrm{~km} \mathrm{~s}^{-1}$ or larger, assuming the travel time to be the typical life time of WR stars. It leads us to suggest that GRB progenitors may be runaway massive stars ejected from compact massive star clusters. The ejection from such super star clusters may lead to a spin-up of these stars, producing the loss of the hydrogen and/or helium envelopes leading to the origin of the type Ibc supernovae associated with GRBs. If this scenario applies to all GRBs, it provides a natural explanation of the very small fraction of massive stars that emit a GRB at the end of their life. An alternative to this scenario could be a binary origin for GRBs, but this still requires an explanation of why it would preferentially occur in low stellar density regions.
\end{abstract}

Key words. cosmology: observations - galaxies: stellar content - galaxies: abundances - stars: Wolf-Rayet - gamma rays: bursts galaxies: general

\section{Introduction}

Gamma-Ray Bursts (GRBs) are believed to trace the death of massive, short lived stars, providing the most energetic events in the Universe. This is further supported by the discovery that several long-duration GRBs are, indeed, associated with the collapse of massive stars to a black hole, referred to as the collapsar model (Galama et al. 1998; Stanek et al. 2003; Hjorth et al. 2003). In this model, a rapidly rotating star undergoing core-collapse produces a jetted GRB along the rotation axis, and blows up the entire star in an energetic supernova explosion (MacFadyen \& Woosley 1999; Klose et al. 2004). The association between GRBs and supernovae ( $\mathrm{SNe}$ ) indicates in many cases that the parent SN population of GRBs is formed by peculiar type Ibc SNe which have been proposed to be SN1988bwlike SNe by Foley et al. (2003). They are characterised by high luminosity peaks $\left(M_{B}\right.$ from -19.5 to -17$)$ and a high expansion velocity of the ejecta $\left(30000 \mathrm{~km} \mathrm{~s}^{-1}\right)$. Nevertheless, other types of SNe (more regular SNIbc or even SNIIn) cannot be excluded.

^ Based on VLT/FORS2 observations collected at the European Southern Observatory, Paranal, Chile, programme No. 073.B-0482(A).

$\star \star$ Also associated to Observatoire de Paris, GEPI, 92195 Meudon, France.
The identity of the progenitors of GRBs has thus still to be addressed. However, while the peculiar SNIbc may be further confirmed as the parent SN population of GRBs, the most favoured progenitors for the collapsar model are the Wolf-Rayet (WR) stars.

Hirschi et al. (2005) have investigated WR stars and have shown that stellar evolution models including detailed effects of rotation predict the conditions for GRB production via collapsars, namely the conditions for black hole formation, the loss of the hydrogen-rich envelope (such as in SNIbc) and enough angular momentum to form an accretion disk around the black hole. Furthermore, if only stars of the particular WO subtype thought to be intimately related to SNIc - are considered, the GRB production rate can fairly well be reproduced. In their model, GRBs are predicted to occur only over a limited metallicity interval at subsolar values (typically at $Z_{\mathrm{SMC}}$ to $Z_{\mathrm{LMC}}$, i.e. $Z \sim 0.2-0.4 Z_{\odot}$ ). However, when magnetic fields are taken into account in these models, it may well be more difficult to produce GRBs (Petrovic et al. 2005). To circumvent this problem Woosley \& Heger (2006) and Yoon \& Langer (2005) suggest rapid rotators at low metallicity (typically $Z \lesssim 0.05 Z_{\odot}$ ) as GRB progenitors, since in these cases a nearly chemically homogeneous evolution and a low stellar mass loss can produce the 
right conditions (high specific angular momentum stars with no hydrogen envelope) for the collapsar model. Establishing a precise metallicity limit, below which this scenario may work, is however difficult due to uncertainties in stellar mass loss rates and initial stellar rotation rates (Yoon \& Langer 2005). To establish observationally the high mass of long-duration GRB progenitors and the collapsar model, and to constrain scenarios like those just mentioned, it is imperative to confirm the presence of WR stars in the region of the GRB, which has never been achieved until now, and to better determine progenitor metallicities.

The first complete optical study of $z<1$ host galaxies has been done by Le Floc'h et al. (2003). It revealed that GRBs occur in galaxies with low luminosities and blue colors (see also Fruchter et al. 1999; Sokolov et al. 2001). In addition, there is growing evidence that the majority of GRB host galaxies are Ly $\alpha$ emitters with star formation rates (SFRs) between 1 to $11 M_{\odot} /$ yr (Fynbo et al. 2003; Jakobsson et al. 2005). All this is indicative of low metallicity environments, as confirmed by direct abundance measurements (Prochaska et al. 2004; Sollerman et al. 2005; Hammer et al., in preparation). As a result, it leads to some controversy, since GRB hosts were formerly believed to be associated with galaxies with strong star formation rates averaging $100 M_{\odot} / \mathrm{yr}$ from radio and sub-mm observations (Berger et al. 2003). It is unclear if the radio emission is simply related to star formation or if the sub-mm detection could be affected by the lack of spatial resolution: this may be questioned by the absence of detection of most GRB hosts by Spitzer (Le Floc'h et al. 2006).

The analysis of the overall spectral energy distribution (SED) shows that the GRB hosts have high specific star formation rates (i.e. high SFRs with respect to their luminosity) and younger stellar populations than an ensemble of all field galaxies (e.g. Christensen et al. 2004). However, as these authors note, the derived ages of 50-200 Myr seem to indicate that GRB hosts are not significantly younger than starburst galaxies at similar redshifts. These "old" ages measured could be the result of large aperture effects or composite stellar populations, i.e. the young population from which the GRB descends might be diluted by older stars.

Further understanding of the GRB production mechanism requires us to study their environments in detail. In this paper we present deep spectroscopic observations of GRB hosts, and focus our analysis on the four most nearby host galaxies: GRB 980425 at $z=0.008$, GRB 031203 at $z=0.1055$, GRB 030329 at $z=0.169$ and GRB 020903 at $z=0.25$. They are bright enough to test for the presence of WR stars, as well as to robustly establish metal abundances through a direct electron temperature measurement. Moreover, they are found to be associated with a type Ibc SN (GRB 980425/SN1998bw: Patat et al. 2001; GRB 031203/SN20031w: Malesani et al. 2004; GRB 030329/SN2003dh: Hjorth et al. 2003; GRB 020903: Soderberg et al. 2005), and when using HST imaging, the SN location can be identified with some accuracy within the host galaxy.

Our VLT observations are described in Sect. 2. The properties of the environments of GRB 980425 and GRB 020903 as deduced from spectroscopy and imaging are discussed in Sect. 3. Based on this data we suggest in Sect. 4 a new scenario for GRB progenitor stars. In Sect. 5 we summarise our result and discuss possible implications. Throughout the paper we adopt the $\Lambda$ CDM cosmological model $\left(H_{0}=70 \mathrm{~km} \mathrm{~s}^{-1} \mathrm{Mpc}^{-1}, \Omega_{\mathrm{M}}=\right.$ 0.3 and $\left.\Omega_{\Lambda}=0.7\right)$.

\section{Observations and measurements}

Spectroscopic VLT/FORS2 observations were done in visitor mode in July 2004 (programme No. 073.B-0482(A)). Among our eight targets, two nearby galaxies were observed, the GRB 980425 at $z=0.0085$ and the GRB 020903 at $z=0.25$ using two differents FORS2 set-ups (600B and 600RI grisms with a resolution $R \sim 1300$ ). For the GRB 980425 host, given the size of the galaxy, the slit was placed as it is shown in Fig. 1. The GRB 980425 host was observed with a total exposure time of $1800 \mathrm{~s}$ and $1500 \mathrm{~s}$ with the 600B and 600RI grisms, respectively, and the GRB 020903 host 7200 s with each grism.

Data reduction and extraction of optical spectra were performed using a set of IRAF procedures developed by our team, which allowed us to reconstruct simultaneously the spectra and the sky counts of the objects. Spectrophotometric calibration of each grism was done using the same star. Broadband filter images were used to compute aperture corrections and check the spectrophotometric calibration.

Flux measurements were performed using the SPLOT package of IRAF. Measurements were performed by two of us (F.H. and H.F.) and compared. In the case of GRB 980245 we also compared our results with those performed on spectra for which the background light of the ESO 184-G82 galaxy had been removed. All results are found to be very similar, which is supported by the remarkable consistency of the derived temperatures using different chemical species. The electron density was derived from the $\mathrm{S}$ II line ratio, the electron temperature from O II $I(3726+3729) / I(7320+7330)$, O III $I(4959+5007) / I(4363)$, $\mathrm{N}$ II $I(6548+6583) / I(5755)$, and S II $I(6716+6731) / I(4068+$ 4076), when available. These estimates were made using the NEBULAR package of IRAF, after correcting the lines for extinction. The package is based on a 5-level atom program and is described by Shaw \& Dufour (1994). Extinction correction was estimated using the $\mathrm{H} \alpha / \mathrm{H} \beta$ ratio, and verified using higher order Balmer lines. All have been found in good agreement, but one, for which we have corrected fluxes from the underlying absorption using the method of Izotov \& Thuan (1999) based on $\mathrm{H} \alpha / \mathrm{H} \beta$ and $\mathrm{H} \gamma / \mathrm{H} \beta$ ratios.

\section{Properties of the environments of GRB 980425 and GRB 020903}

We now discuss our FORS2 spectra of the SN1998bw remnant and several nearby regions of the GRB 980425 host as well as HST imaging of this galaxy, and FORS2 spectroscopy and HST imaging of the more distant GRB 020903. A brief description of the hosts of GRB 030329 and GRB 031203 (based on archival data) is given for comparison in Sect. 4.

\subsection{GRB 980425 host galaxy}

Figure 1 displays the slit position with labels for the regions around SN1998bw. HST/STIS images (ESO/HST archive programmes 8640,8243 , and 8648) of two regions are shown, revealing 7 sub-areas in the immediate vicinity of SN1998bw (Fynbo et al. 2000), and the "WR region" includes a very luminous main component, hardly resolved $(0.03 \operatorname{arcsec} F W H M)$, corresponding to 5 pc FWHM.

\subsubsection{A bright and compact HII region with WR stars}

This region lies approximately 0.8 kpc NW from the SN1998bw, and its spectrum presents numerous emission lines, with a broad 


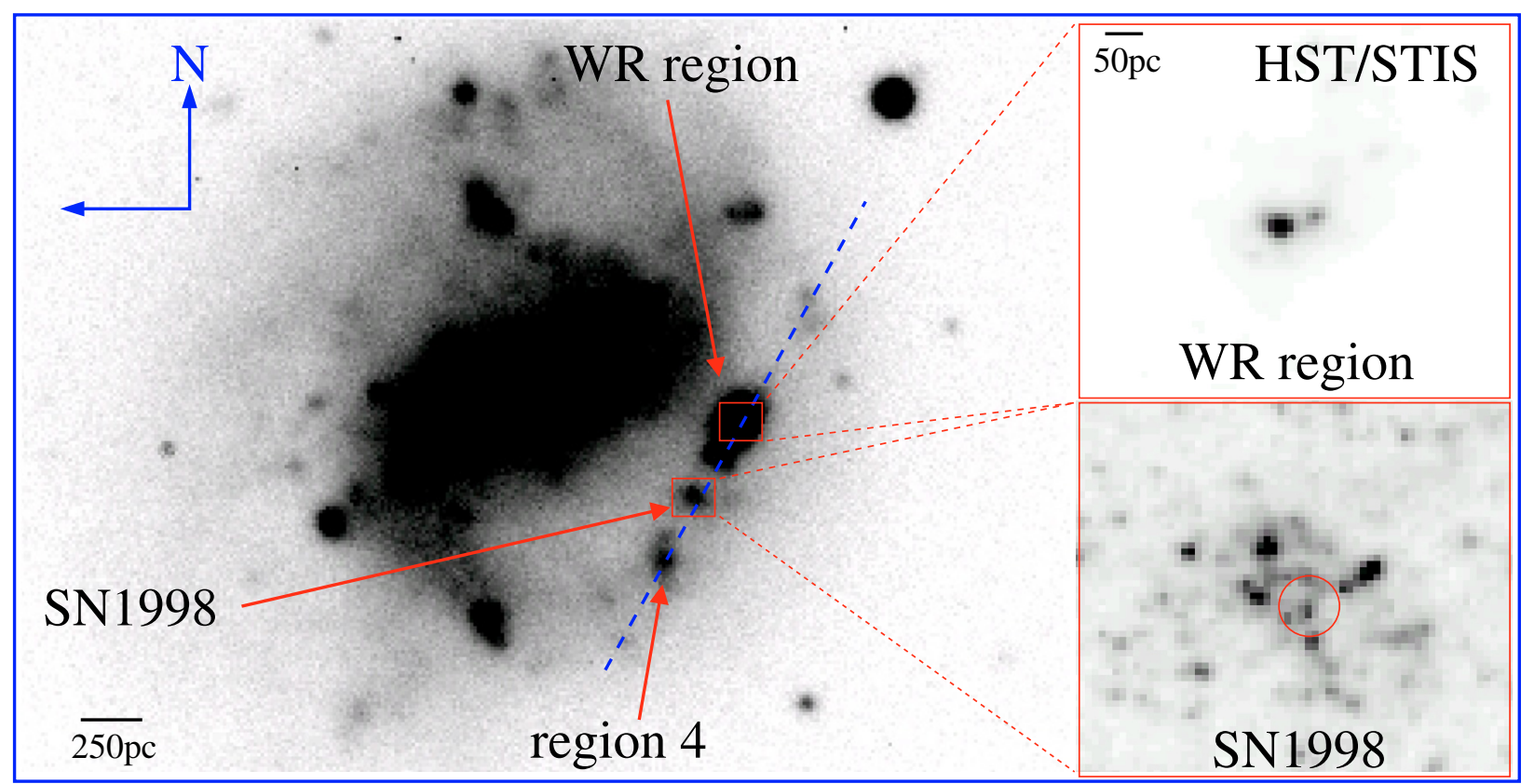

Fig. 1. VLT image of the ESO-184-G82 galaxy showing the slit location and the regions surrounding the SN1998bw remnant. On the right, enlargement of the two area (WR region on top, SN region on bottom) based on HST/STIS imagery. The location of the SN is indicated by a circle.
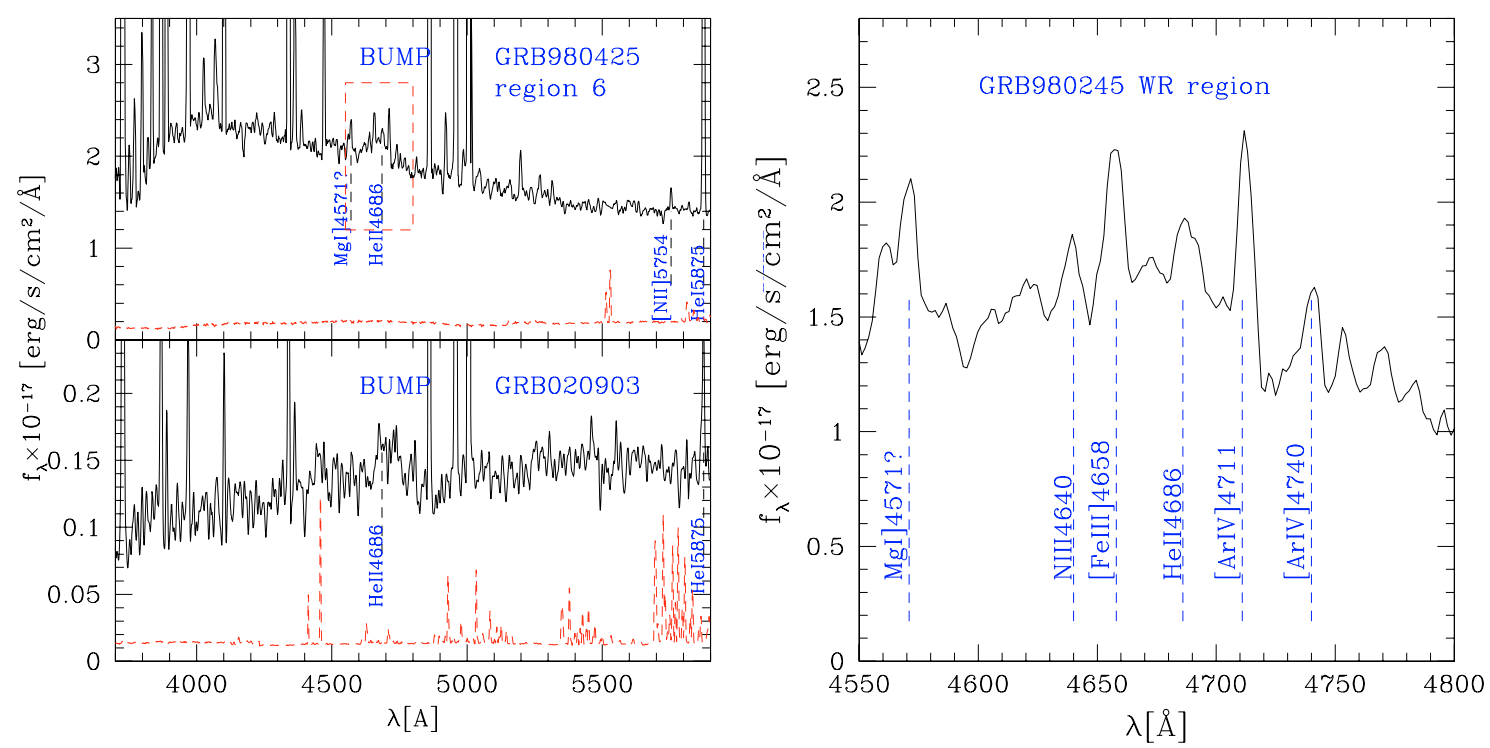

Fig. 2. Spectra of the WR region of the GRB980425 host (top, left) and of the GRB020903 host (bottom, left). On the right is shown an enlargement around HeII 4686 of the spectrum of the WR region of the GRB980425 host.

range of chemical species (see Fig. 2 and Table 1). Its density, temperature and oxygen abundance (see Table 2) are characteristic of a vigorous star forming region with $Z \sim 0.5 Z_{\odot}$ (assuming $12+\log (\mathrm{O} / \mathrm{H})=8.69$ for the solar abundance, see Allende Prieto et al. 2001). $\mathrm{H} \alpha$ and $\mathrm{H} \beta$ show very large equivalent widths, 1400 and $200 \AA$, respectively. After correcting for the aperture effect (factor 4.6) and for extinction, we find a luminosity of $1.24 \times 10^{40} \mathrm{erg} \mathrm{s}^{-1}$ in the $\mathrm{H} \beta$ line, which corresponds to 2300 equivalent O7V stars (cf. Schaerer \& Vacca 1998).

The spectrum shows a prominent blue WR bump around the He II 4686 line, with many faint emission lines (ArIV 4711, 4740, NIII 4640, and FeIII 4658, see Fig. 2). The absence of carbon lines (CIII 5696 and CIV 5808) suggests that most of the WR stars are of the WN type. Using the luminosity of the blue bump from 4650 to $4686 \AA$ (see Schaerer \& Vacca 1998), we estimate the number of WN stars to be $86 \pm 20(1 \sigma$ error estimated from the continuum) in that region (assuming WN7 star luminosities). It agrees with the measurement of the faint HeII4686 line (see Table 1), which corresponds to the emission of 46 to $92 \mathrm{WN}$ stars. The large $\mathrm{H} \alpha$ and $\mathrm{H} \beta$ equivalent widths, the presence of WR stars, and fits to the spectral energy distribution are all indicative of a very young ( $\sim 1-6 \mathrm{Myr}$, depending on the age indicator used) massive star forming region. These properties, as well as the size of the region (O'Connell et al. 1994), show that we are dealing with a (possibly multiple?) massive, young, super star cluster.

We also notice the possible presence of $\mathrm{MgI}$ ] 4571 in emission, a feature commonly seen in the nebular phase of type Ibc SNe (see Patat et al. 2001; Foley et al. 2003) and broad wings in the bottom of the $\mathrm{H} \alpha$ emission line revealing gas 
Table 1. Flux measurements of the GRB host galaxies.

\begin{tabular}{lrrrr}
\hline \hline & \multicolumn{3}{c}{ GRB 980425 } & GRB 020903 \\
& SN reg. & WR reg. & Reg. 4 & \\
\hline O II $\lambda 3727$ & 4.020 & 44.00 & 2.680 & 6.89 \\
Ne III $\lambda 3869$ & 0.550 & 11.30 & 0.055 & 2.05 \\
O III $\lambda 4363$ & $<0.030$ & 1.55 & $<0.030$ & 0.54 \\
N III $\lambda 4640$ & - & 0.30 & - & 0.0078 \\
He II $\lambda 4686$ & $<0.052$ & 0.53 & - & 0.026 \\
Ar IV $\lambda 4711$ & - & 0.34 & - & 0.12 \\
Ar IV $\lambda 4740$ & - & 0.16 & - & 0.20 \\
H $\beta$ & 0.910 & 35.40 & 0.230 & 4.40 \\
O III $\lambda 4959$ & 0.900 & 49.70 & 0.570 & 7.40 \\
O III $\lambda 5007$ & 2.040 & 201.20 & 1.100 & 33.50 \\
N II $\lambda 5755$ & - & 0.17 & - & - \\
O I $\lambda 6300$ & 0.400 & 1.70 & 0.200 & 0.65 \\
S II $\lambda 6312$ & 0.040 & 0.90 & - & - \\
N II $\lambda 6548$ & 0.380 & 4.17 & 0.340 & 0.25 \\
H $\alpha$ & 3.860 & 183.90 & 1.890 & 16.80 \\
N II $\lambda 6583$ & 0.880 & 11.30 & 0.520 & 0.72 \\
S II $\lambda 6716$ & 0.600 & 10.90 & 0.540 & 2.39 \\
S II $\lambda 6731$ & 0.840 & 8.60 & 0.590 & 1.71 \\
Ar III $\lambda 7136$ & 0.190 & 7.90 & 0.096 & - \\
O II $\lambda 7325$ & 0.195 & 3.20 & 0.126 & - \\
Ar II $\lambda 7751$ & 0.03 & 2.08 & - & - \\
\hline
\end{tabular}

Notes: Measured fluxes before aperture correction in $10^{-16} \times$ $\operatorname{erg}^{-1} \mathrm{~cm}^{-2} \AA^{-1}$. Typical errors $(1 \sigma)$ are 0.03 for the faint lines as estimated from the SPLOT routine.

motion at velocities up to $3000 \mathrm{~km} \mathrm{~s}^{-1}$. If real, these features, combined with the large number of WR stars, might indicate the presence of recent supernovae in this region.

While the location of SN1998bw is beyond doubt (see Galama et al. 1999), its association with GRB 980425 has been much debated. The major argument in favour of the association of the two events is their remarkable temporal coincidence. The fact that other SN of the same type as SN1998bw have been found a few weeks after GRB events (see e.g. Foley et al. 2003) is also a good argument to support the association. Nevertheless, the peculiar properties of the WR region near the $\mathrm{SN}$ has prompted us to investigate whether some supernovae might have occurred there few years before our observations. We have used the HST/STIS imagery (see Fynbo et al. 2000) taken at three different epochs, namely June and November 2000 and August 2001. Special care has been taken to derive a common astrometric solution for each image and we derive a 0.03 STIS pixel (or 0.75 mas) accuracy using three stars around the WR and SN regions. While the variability of SN1998bw is well detected, we found no indication of variability in the WR region. We notice, however, that the detection of a faint signal in a very bright area is prevented by the Poisson noise of the total signal, and so we would not be able to see a faint signal such as the SN1998bw remnant two years after its maximum, if it was embedded in the bright WR region. However, we believe that a strong variability similar to that of SN1998bw at its peak would have been detected in the WR region, since this field has been thoroughly surveyed since May 1998. Recall that the SN1998bw luminosity near its maximum surpasses the luminosity of the WR region (see Fig. 2 of Galama et al. 1999). Hence, we conclude that the association of the GRB 980425 with SN1998bw is very probable, and that the WR region is a strong star-forming region including a very bright (possibly multiple) super star cluster separated by $800 \mathrm{pc}$ from the SN.
Table 2. Physical properties of the GRB host galaxies.

\begin{tabular}{lrrrr}
\hline \hline & \multicolumn{3}{c}{ GRB 980425 } & \multicolumn{2}{c}{ GRB 020903 } \\
& Region SN & Region WR & Region 4 & \\
\hline$N_{\mathrm{e}}[\mathrm{S} \mathrm{II}]^{a}$ & 27 & 158 & 498 & 27 \\
$T_{\mathrm{e}}[\mathrm{O} \mathrm{II}]^{b}$ & 11939 & 11129 & 9160 & - \\
$T_{\mathrm{e}}[\mathrm{O} \mathrm{III}]^{b}$ & $<13966$ & 11900 & $<18274$ & 14852 \\
$T_{\mathrm{e}}[\mathrm{N} \mathrm{II}]^{b}$ & - & 11249 & - & - \\
$12+\log (\mathrm{O} / \mathrm{H})]^{c}$ & 8.25 & 8.39 & 9.00 & 7.97 \\
$\mathrm{~N} / \mathrm{O}$ & 0.21 & 0.043 & - & 0.013 \\
$A_{\mathrm{V}}{ }^{c}$ & 1.02 & 1.51 & 0.98 & 0.76 \\
\hline
\end{tabular}

Notes: ${ }^{a}$ Electronic density estimated from the $\mathrm{S}$ II flux ratio $I(6716) / I(6731){ }^{b}$ Temperature in $\mathrm{K}$ estimated from the following flux ratios: O II $I(3726+3729) / I(7320+7330)$, O III $I(4959+$ $5007) / I(4363), \mathrm{N}$ II $I(6548+6583) / I(5755)$, and S II $I(6716+$ 6731)/I(4068 + 4076). ${ }^{c}$ Metallicity, $12+\log (\mathrm{O} / \mathrm{H})$, estimated using the effective temperature method. ${ }^{d}$ Extinction coefficient, $A_{\mathrm{V}}$ (in magnitudes), has been computed using the standard Balmer ratio of $\mathrm{H} \beta$ and $\mathrm{H} \alpha$.

The properties of this cluster are indeed interesting, and sufficiently strong to explain reasonably well the detection at $24 \mu \mathrm{m}$ reported by Le Floc'h et al. (2006). Indeed, assuming standard SFR conversion factors, the star formation rate derived from the $\mathrm{H} \alpha$ line is $0.34 M_{\odot} / \mathrm{yr}$ (aperture and extinction corrected), a value in excellent agreement with what can be inferred from its IR luminosity $\left(L_{\mathrm{IR}}=2 \times 10^{9} L_{\odot}\right.$, see Le Floc'h et al. 2006), i.e. $S F R=0.35 M_{\odot} / y r$. From its absolute scales the properties of the WR region are also similar to those of the embedded super star cluster ("supernebula") in the nearby starburst NGC 5253. The bolometric luminosity of the dominant embedded cluster in NGC 5253 is $L_{\text {bol }} \sim(1-3) \times 10^{9} L_{\odot}$ (Beck et al. 1996; Vanzi \& Sauvage 2004), and it contains several thousand O stars within a small (pc or even sub-pc scale) region (e.g. Turner et al. 2003). However, the extinction in the WR region of the GRB 980425 host is much smaller. Further comparisons are beyond the scope of this paper.

\subsubsection{The region surrounding the SN1998bw remnant}

This region is almost 10 times fainter than the WR region, and provides a spectrum with a lower $\mathrm{S} / \mathrm{N}$. Nevertheless, it shows enough emission lines to derive a full diagnosis of its interstellar medium. It shows a more moderate extinction and with $Z \sim 0.36 Z_{\odot}$ a somewhat lower oxygen abundance than the WR region and region 4 (see Table 2). Interestingly, and in contrast to the WR region, this region also has a high nitrogen over oxygen abundance of $\mathrm{N} / \mathrm{O}=0.24$, almost twice the solar value. While such a high N/O ratio is approximately 4 times larger than that found in HII regions (cf. e.g. Liang et al. 2005; Izotov et al. 2006), such strong $N$ line intensities can be found in SN remnants (Smith et al. 1993). This $\mathrm{N}$ excess could be related to the enrichment from the progenitor star of SN1998bw, as expected from rotating stars. For example, similar N/O ratios are predicted by the yields of $\sim 10-20 M_{\odot}$ stars in the rotating stellar evolution models of Hirschi et al. (2005) albeit at somewhat larger metallicity than the one observed for this SN region. However, dilution with pre-existing ISM will reduce the resulting N/O ratio.

The $\mathrm{SN}$ region shows moderate $\mathrm{H} \alpha$ and $\mathrm{H} \beta$ equivalent widths of 88 and $16 \AA$, respectively, indicative of ages of $\sim 6-8 \mathrm{Myr}$ adopting our evolutionary synthesis models. The aperture (factor 3.27) and extinction corrected $\mathrm{H} \beta$ flux corresponds to only $\sim 10 \mathrm{O} 7 \mathrm{~V}$ equivalent stars. We do not detect the presence of WR stars, which cannot only be attributed to the relatively 


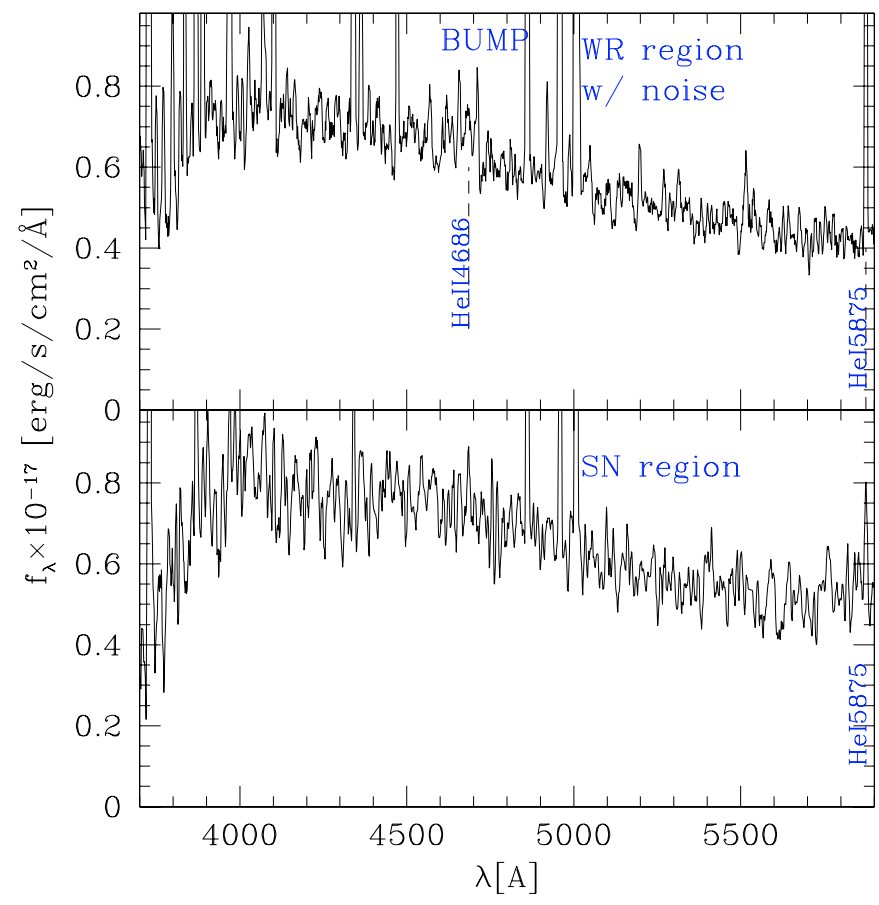

Fig. 3. Spectrum of the SN1998bw remnant region (bottom) compared to the scaled spectrum (top) of the WR region at the same $\mathrm{S} / \mathrm{N}$ of the GRB 980425 host. It shows that the absence of WR features in the SN1998bw remnant cannot be related simply to the lower S/N.

low $\mathrm{S} / \mathrm{N}$ of the spectrum. Indeed, Fig. 3 compares the spectrum of the $\mathrm{SN}$ region with that of the WR region if it was observed at the same $\mathrm{S} / \mathrm{N}$. The derived upper limit on the luminosity of the HeII4686 line (see Table 1) indicates fewer than or 1 WR star in that region. This is not surprising given the small number of massive stars and the relatively low WR/O star ratio, WR/O 0.05 , observed in the WR region. Recall also that HST/STIS (see Fig. 1) is able to resolve the $\mathrm{SN}$ region in 7 small sub-areas, and that the brightness at the precise location of the SN is small compared to other sub-areas. According to the photometry of Fynbo et al. (2000), in April 2000, the SN contributed to only $13 \%$ of the $V$ luminosity of the region. Our spectroscopic observations were made 3 years after the STIS observations and the SN remnant probably has faded away since April 2000: it is probable that no massive stars are present in the SN sub-region.

\subsection{GRB 020903 host galaxy}

The GRB occurred in the outskirts of a small, very irregular galaxy at $z=0.25$ with $M_{B}(\mathrm{AB})=-19.3$ (see Fig. 4). The slit was centered on the position of the reported afterglow (see Soderberg et al. 2004a). GRB 020903 presents many similarities with GRB 980425. Indeed, Soderberg et al. (2005) have convincingly shown that the event was followed by a supernova of the same type as SN1998bw. The residual HST image in Fig. 4 (top-right) shows the precise location of the $\mathrm{SN}$, which has occurred at a 0.115 arcsec offset from a compact, unresolved region, in the outskirts of the host galaxy. At the distance of the host galaxy $(z=0.25)$, this offset corresponds to $460 \pm 100 \mathrm{pc}$, a value close to the distance between the WR and $\mathrm{SN}$ regions in the GRB 980425 galaxy.

The spectrum at the position of GRB 020903 (see Fig. 2) reveals an active star forming region, with a strong oxygen deficiency of $Z=0.19 Z_{\odot} . \mathrm{H} \alpha$ and $\mathrm{H} \beta$ show large (rest-frame)

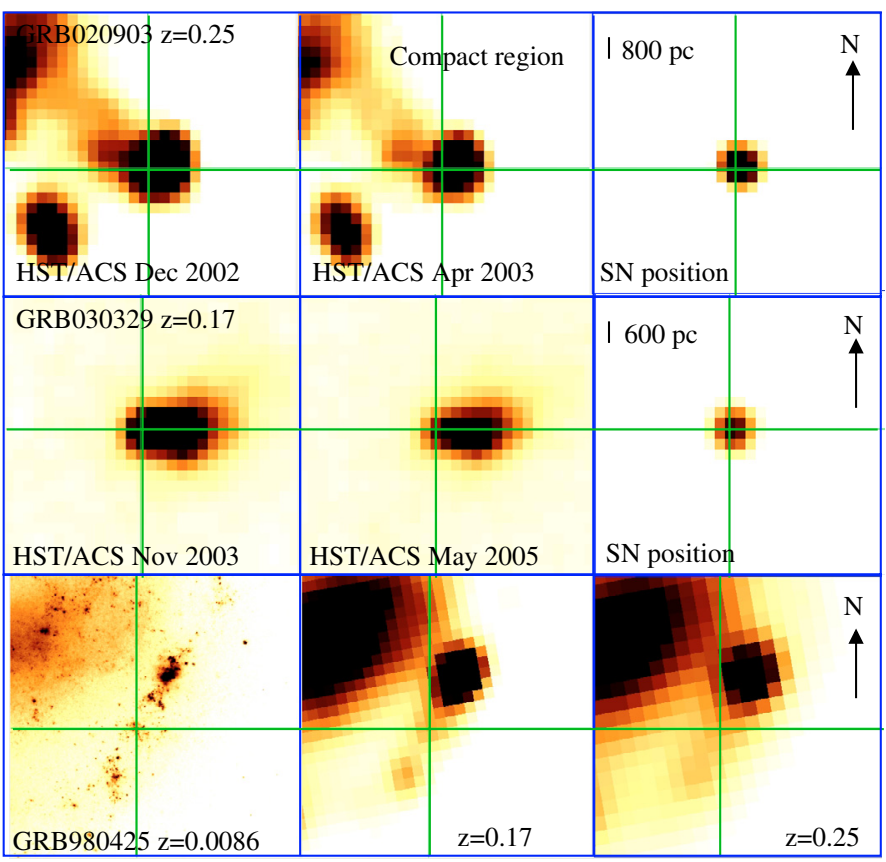

Fig. 4. Top panels: HST/ACS/F606W image taken 91 (left) and 300 (middle) days after the GRB 020903 and showing the associated SN (same type as SN1998bw, see Soderberg et al. 2005). The image on the right shows the residual derived from our extraction, and confirms the result of Soderberg et al. The SN (peak identified with the light cross) exploded $460 \mathrm{pc}$ off a luminous region, which is not resolved even at the resolution of the ACS. Middle panels: HST/ACS/F606W image taken 8 (left) and 25 (middle) months after the GRB 030329 event and showing the associated SN (right, light cross). Bottom panels: GRB 980425 image at $z=0.0086$ (left) plotted as it would be seen if it was at the redshift of GRB 030329 (middle) and of GRB 020903 (right). This illustrates that the offset between the SN and WR regions in GRB 980425 is similar to that observed in the two more distant GRBs.

equivalent widths, 245 and $40 \AA$, respectively. These values are intermediate between those of the SN and WR regions of the GRB 980425 host. This is not surprising, knowing that the slit has likely included several regions around the GRB location. After correcting for extinction, we find a luminosity of $6.9 \times$ $10^{39} \mathrm{erg} \mathrm{s}^{-1}$ in the $\mathrm{H} \beta$ line, which can be interpreted as produced by $\sim 1300 \mathrm{O}$ stars (assuming a O7V stellar type) present in the aperture. A larger value might be inferred if the aperture correction (factor 6) was applied.

Figure 2 shows a strong signature of a blue WR bump and using the HeII4686 emission line, we infer a WNL/O ratio ranging from 0.14 to 0.2 (approximately $\$ 200$ WR stars). The blue bump does not show the same features as that of the GRB 980425 WR region. This is not so surprising after examining the large variety of blue bump features from a survey of WR galaxies (Guseva et al. 2000). The WR bumps are made of blends of a large variety of emission lines and can thus be subject to variations from one galaxy to another. The relatively low $\mathrm{S} / \mathrm{N}$ of our spectrum can also alter the appearance of the blue bump. It is more difficult to assess the age of the stars near the location of the GRB, because the slit likely includes components from other regions of the galaxy. Assuming the extinction $\left(A_{\mathrm{V}}=0.76\right)$ derived from the ionised gas, the fit of the spectral energy distribution reveals the need for an older stellar population.

In summary, the properties of the GRB 020903 host galaxy seem to be very similar to those of the GRB 980425 host, except that it shows a higher electron temperature and a smaller 
oxygen abundance. As for GRB 980425, the supernova associated with GRB 020903 seems to have occurred at several hundred parsecs from a bright, relatively compact region, which is most likely responsible for the numerous WR and $\mathrm{O}$ stars seen in our spectrum.

\section{Discussion}

The proximity of the two GRB host galaxies (980425 and 020903) has allowed us to detail at an unprecedented level their spatial and spectral properties. However, these two GRB events are among the least energetic GRBs (see Soderberg et al. 2004b), and in the following discussion one should be cautious before extrapolating their properties to the numerous cosmological GRBs observed at large distances. However, it has been argued that sub-energetic bursts are simply events viewed away from the jet axis. Indeed, Guetta et al. (2004) have shown that this interpretation matches the statistics of both low energetic GRBs at low redshifts and high energetic GRBs at cosmological distances. Fynbo et al. (2004) also argue for such a case for the X-ray flash XRF 030723.

\subsection{Inferences on the connection between GRB and star formation}

GRBs occur in galaxies that are generally sub- $L^{*}$ and even dwarfs (see Le Floc'h et al. 2003). The spectra of their hosts include a large variety of emission lines, including most of the Balmer series and Helium lines. This is characteristic of a very young burst, which is further supported by the fact that few Myr old stars (including WR stars) dominate the continuum. Within these small galaxies, we find that GRBs (and their associated supernovae) do not occur in regions containing a large number of massive stars. This is in strong contrast to simple expectations from the massive star collapsar model, where the GRB progenitor star is thought to represent a rare case among a large number of massive stars.

In both cases studied here, we find that the GRB occurred at distances of $\sim 400$ to $800 \mathrm{pc}$ from a compact and luminous region, which is just resolved ( $5 \mathrm{pc} F W H M$ ) in the case of the WR region in the GRB 980425 host. In other words, GRBs neither occur directly in massive star forming regions nor in massive LIRGs (see Le Floc'h et al. 2006), but in regions that show very few or no massive stars (e.g. the $\mathrm{SN}$ region in the GRB 980425 host). This, together with the generally sub-solar metallicities measured in GRB hosts casts serious doubt on the direct relation between GRBs and star formation, which is dominated by stellar formation in massive $(1<z<3$, see Caputi et al. 2005) or in intermediate massive $(0.4<z<1$, see Hammer et al. 2005) galaxies. However, as discussed below, our finding of a close spatial association and possibly a dynamic connection between GRBs and a "nearby" super star cluster or a massive star-forming region, suggests a more indirect connection between GRB and star formation.

\subsection{Inferences on GRB progenitors}

What mechanisms can explain these powerful events? Recall that the collapsar model favours a WR origin for the GRB (Woosley et al. 1999; Meszaros 2002). This is supported by the specific nature of the associated supernovae, whose type (SNIb or c) indicates that they have lost their hydrogen and/or helium envelope, as is expected if they originate from WR stars (e.g.

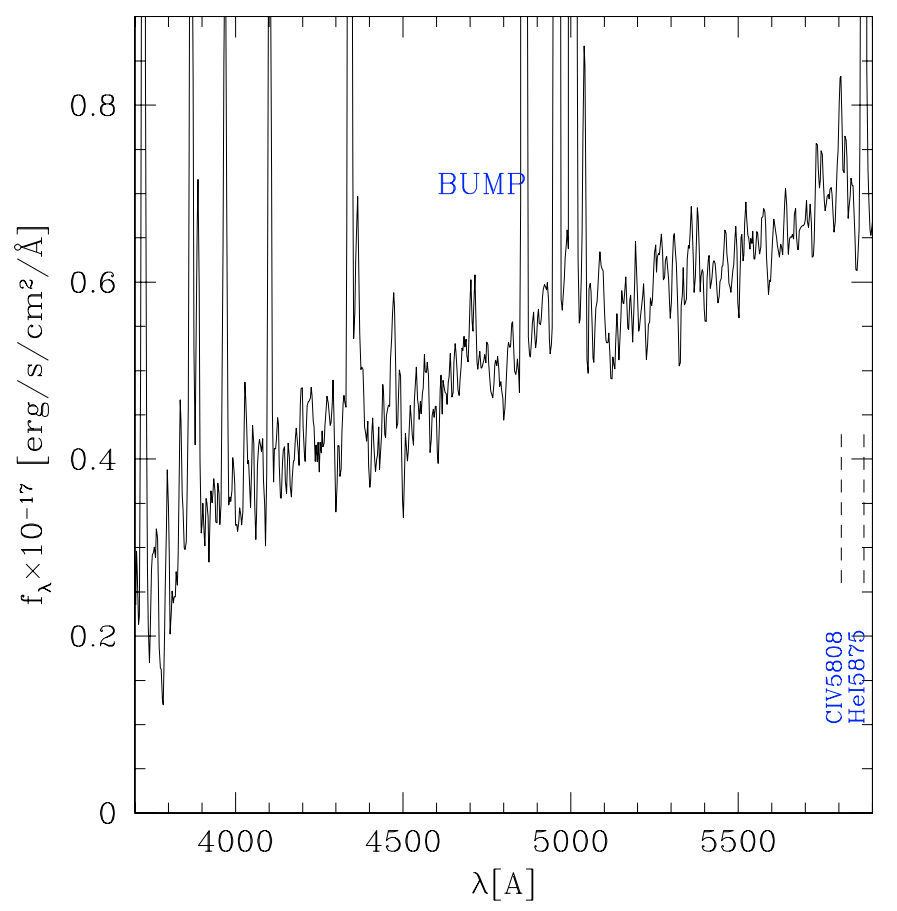

Fig. 5. Spectrum of the GRB 031203 host $(2 \times 1800$ s $)$ taken in September 2004 using the $300 \mathrm{~V}$ grism with VLT/FORS2 (from ESO archive, programme No. 073.D-0255(A)). The red slope of the energy distribution can be attributed to the low Galactic declination ( $b^{I I}=4.7$ ) leading to an extinction $A_{\mathrm{V}}=3.62$ (see Prochaska et al. 2004). A blue bump around the HeII 4686 line is detected as well as a bump around the CIV 5808 line, confirming the presence of WR stars in the host galaxy.

Hirschi et al. 2005). This is consistent with the strong nitrogen excess we observe in the SN1988bw remnant region, which may be produced by the progenitor wind or the $\mathrm{SN}$ of an initially fast rotating star (see Meynet \& Maeder 2002).

Are WR stars found in all GRB host galaxies? We have investigated archival data from ESO telescopes, to acquire good spectra of the most nearby GRB host galaxies, i.e. those for which 8 meter class telescopes are able to detect the faint signature of this stellar population. Considering the four similar objects pointed out by Soderberg et al. (2004b, see their Fig. 2), this includes our two targets plus GRB $031203(z=0.1055)$ and GRB $030329(z=0.169)$. Altogether these objects represent the low energetic end of GRBs, and for all of them, supernovae have been observed few weeks after the GRB. Nevertheless, GRB 020903, GRB 030329, and GRB 031203 are almost 100 times brighter than GRB 980425 and are intermediate between this event and cosmological GRBs. Unfortunately available VLT data on the GRB 030329 host are of insufficient quality $^{1}$. On the other hand, the spectrum of the GRB 031203 host has a quality similar to our spectrum of GRB 020903. Figure 5 shows its spectrum and we can see that a blue bump around the HeII 4686 line and also a bump around the CIV 5808 line are unambiguously detected, indicating the presence of WR features, respectively of the WNL and WC stars. For all the observed GRB hosts having a spectrum with enough quality to detect WR stars, we indeed detect WR stars.

\footnotetext{
${ }^{1}$ We extracted deep observations made at VLT in excellent seeing conditions ( 0.5 arcsec); unfortunately this very compact object has been observed with a wide slit (1.3 arcsec) and the region near the bump is highly contaminated by residuals of the strong OI 5577 sky line.
} 
Our observations of the WR region of the GRB 980425 host show a very compact ( 5 pc $F W H M$ ) super star cluster containing thousands of $\mathrm{O}$ stars and almost one hundred WR stars. The bright region in GRB 020903 separated by a projected distance of $\sim 460 \mathrm{pc}$ from the SN (see Fig. 4) is probably responsible for at least a part of the observed WR emission. It also includes a compact component, apparently not resolved by the ACS camera and therefore smaller than $100 \mathrm{pc}$ at $z=0.25$. Unfortunately there is no HST imagery of the GRB 031203 host, and the precise location of the GRB/SN is still unclear from ground-based data (GRB from 0.2 to 1 arcsec off the host galaxy center according to Gam-Yan et al. 2004 or to Prochaska et al. 2004). Images of the GRB 030329 host (see Fruchter et al. 2003) reveal a very faint host galaxy $\left(M_{V}=-16.5\right)$ and the $\mathrm{SN}$ is found at its edge, off by $750 \mathrm{pc}$ from the galaxy center (see Fig. 4). The offsets revealed by Figs. 1 and 4 are very robust because they are based on HST images taken at different epochs revealing the SN, and the accuracy is better than half an ACS pixel (0.05 arcsec). They do not differ much from that found by Bloom et al. (2002) on a larger sample of GRBs (median value of $1.3 \mathrm{kpc}$ for the offset), although their result is less accurate because it is based on a comparison between HST and ground based images. Thus it appears that GRBs often (or always?) occur in the outskirts of regions populated by hot and massive stars including WR stars.

Based on this new information on the spatial location and the stellar populations we now discuss the possible scenarios for GRB progenitors. Assuming that GRB 980425 is a prototype of other GRBs discussed in this paper, we are left with two hypotheses: either the GRB progenitor is born in a low stellar density region (in situ hypothesis) or it has been expelled from high density stellar regions (runaway hypothesis).

\subsection{GRB as runaway, fast rotating massive stars expelled from superstellar clusters?}

If GRB hosts include clusters with WR stars in large numbers, why does the SN (and then the GRB) occur several hundreds of parsec away from the WR region? Assuming this is not a coincidence we suggest the following "runaway GRB" scenario, which may be essential to achieve the necessary conditions for the formation of a GRB. Within the high stellar density of massive super star clusters, some stars (or double stars) can be ejected dynamically after one or more elastic collision or from supernova kicks in binary systems. During such a collision, the star may have acquired a very large angular momentum, enough to lose its hydrogen or even helium envelope providing, typically 3 Myr later, a progenitor of a SNIbc such as those observed after the GRB event.

In the case of GRB 980425, for the progenitor to travel $800 \mathrm{pc}$ from the WR region to the SN1998bw region in say 3-6 Myr, a velocity of $\sim 260-130 \mathrm{~km} \mathrm{~s}^{-1}$ is necessary. Such velocities are somewhat larger than (but not unseen) the typical velocity of Galactic $\mathrm{O}$ to early B-type runaway stars, which are thought to be runaway stars from stellar clusters (see e.g. Blaauw 1993; Tenjes et al. 2001; de Wit et al. 2005). This leaves this possibility for sufficiently young, i.e. massive GRB progenitors.

We do not suggest that all massive runaway stars give rise to a GRB, as this would correspond to $\sim 10-15 \%$ of all O stars, based on the knowledge of Galactic runaway stars (Gies 1987; de Wit et al. 2005). However, in the case of the very massive and compact super star clusters observed in the GRB host galaxies, the dynamical conditions, ejection probabilities, and the resulting properties of runaway stars may be quite different from the less dense and less populated associations and clusters typically found in our Galaxy (see e.g. Leonard \& Duncan 1990; Leonard 1991; Portegies Zwart et al. 1999). Furthermore, only the runaway stars with some peculiar ejection history and high angular momentum may produce a GRB. An interesting case possibly resembling our suggested runaway stars is the high velocity star HIP 60350 thought to be dynamically ejected from the compact region NGC 3603 (Tenjes et al. 2001). However, this object is of spectral type B3-4V, i.e. a priori of too low mass to produce a collapsar via "normal" single star evolution. Hobbs et al. (2005) also find large velocities for the proper motions of 233 pulsars with mean 1D speeds of $152 \mathrm{~km} \mathrm{~s}^{-1}$ $\left(\sigma=265 \mathrm{~km} \mathrm{~s}^{-1}\right)$.

Runaway stars can be produced either by supernova explosions in massive close binary system or via strong dynamical interactions in young star clusters (Blaauw 1993). It is beyond the scope of this paper to differentiate between these alternatives. Portegies Zwart et al. (1999) have simulated the "ecology" of a similar system, the central R136 cluster of 30 Doradus in the Large Magellanic Cloud. They found that physical collisions between stars are quite frequent, and closely linked with the evolution of the star cluster. Portegies Zwart et al. (1999; see also Leonard 1995) argue that dynamically ejected runaway stars can be massive and could have acquired a large angular momentum which might be consistent with expectations for a SNIbc progenitor. Bally \& Zinnecker (2005) suggest that the merger of two massive stars within dense clusters could be a pathway leading to hypernovae/GRBs. Combining our empirical findings with their models suggests that GRBs originate from (very) rapidly rotating stars - resulting from previous stellar collisions in the cluster core - which are ejected during a subsequent dynamical interaction. Such dynamical interactions might also help to alleviate the difficulties to form GRBs in rotating stellar models including the breaking by magnetic fields found in particular at solar or somewhat subsolar metallicities (Petrovic et al. 2005; Woosley \& Heger 2006; Yoon \& Langer 2005). Most likely the runaway GRB progenitor ejected from the cluster is a single star or a tight binary.

This scenario has several advantages. It explains (by construction) the observed spatial shift between the GRB position and a nearby massive star-forming region, as observed for GRB 980425 and also tentatively indicated for GRB 030329 and GRB 020903. It accounts for the fact that all our studied GRB host galaxies are indeed WR galaxies. Furthermore, it allows us to reconcile single star collapsar models with the lack of massive stars observed in the immediate vicinity of GRB 980425. Indeed, a rather low branching ratio of GRB/SNIbc (typically $R=N(\mathrm{GRBSNe}) / N(\mathrm{SNIbc}) \sim$ (2-4) $\times 10^{-3}$, van Putten 2004; Podsiadlowski et al. 2004) implies that statistically a GRB should be accompanied by a large number of massive stars, which are not found in this region. In other words, if GRBs result from the tail of a distribution of properties of massive single stars, the population corresponding to the remainder of this distribution, i.e. several thousand massive stars, should be present. The age and the metallicity of the super star cluster (SSC) is compatible with massive single star progenitor models (e.g. Hirschi et al. 2005).

On the other hand, if the progenitor of SN1998bw was ejected from the nearby SSC ("WR region" in Fig. 1) in a random direction, why is it observed within a small, but relatively inconspicuous region surrounded by 6 point sources (see Fig. 1; and Fynbo et al. 2000)? Using Fig. 1 we have tested if this spatial association is purely fortuitous, assuming that the GRB progenitor is a runaway star expelled from the WR region. We have drawn a circular annulus centered on the WR region with an 
internal radius of 0.55 arcsec (to exclude the whole WR region) and an external radius of 5.3 arcsec (to include the $\mathrm{SN}$ region); it corresponds to an area of $87.3 \operatorname{arcsec}^{2}$. Assuming that the runaway star has been expelled in a random direction, we calculate the probability of the association of GRB 980425 with the SN region. We find that $9.6 \operatorname{arcsec}^{2}$ among $87.3 \operatorname{arcsec}^{2}$ correspond to pixels with larger surface brightness than the $\mathrm{SN}$ region, i.e. to denser stellar regions. It leads to a marginal probability (11\%) of finding an expelled runaway star in a region as bright or brighter than the SN region. A similar but more complex estimate can be attempted by accounting for all the point sources found in the corresponding area. It is, however, limited by several bright regions which could not be resolved into individual sources. The number of point sources in the $87.3 \operatorname{arcsec}^{2}$ area ranges from 250 and 300 . The probability of finding the expelled star surrounded by 6 point sources within $1 \operatorname{arcsec}^{2}$ ranges from 7 to $13 \%$. We conclude that the location of the $\mathrm{SN}$ is marginally fortuitous and thus not unexpected.

\subsection{GRBs from binaries occurring in situ within low stellar density regions?}

If the GRB progenitor was born in a low density region, it is rather implausible that it is a single, fast rotating, massive star. Alternatively, several binary scenarios have been proposed for the formation of long duration GRBs (e.g. Fryer \& Woosley 1999). Ages of $\sim 6-8 \mathrm{Myr}$ (see Sect. 3.1.2) are a priori compatible with binary collapsar or He star merger models (see Fryer \& Woosley 1999).

A (massive) binary scenario does not require a very massive, rich cluster or region, as the low GRB/SNIbc branching ratio might e.g. be explained by processes related to the nucleation of black holes (van Putten 2004). However, there is no reason why in this case GRBs should be found preferentially in low stellar density regions (e.g. less than $10 \mathrm{O}$ stars in the $\mathrm{SN}$ region of GRB 980425). When considering larger samples, one should then find GRBs spatially distributed proportionally to the number of massive stars, i.e. to current star formation.

\subsection{Further observational tests}

Our observations and the two scenarios discussed above imply that the "classical" single star collapsar model has to be abandoned for a binary scenario or for our newly suggested "runaway ejection scenario" (or a combination of both). What additional and possibly decisive tests distinguishing these scenarios can be envisaged?

The most decisive test of the runaway ejection scenario would be the localisation of more isolated GRBs together with a "parent" super star cluster from which they were ejected. The lack of a fairly massive and young cluster in reasonable proximity of the GRB would exclude our scenario. We are also puzzled by the fact that, at least in two cases (GRB 980425 and GRB 030329, see Fig. 4), the GRB/SNs seem to be located in the prolongation of the elongated WR regions. Is this consistent with a runaway scenario? Such investigations require the detection of many nearby GRBs, preferentially at $z<0.05$, which is challenging.

Other potentially interesting constraints may come from the study of absorption lines originating from the burst environment (cf. Schaefer et al. 2003; Mirabal et al. 2003; Starling et al. 2005; Berger et al. 2006; Prochaska et al. 2006). For instance, narrow Fe II* and Si II* fine structure absorption lines have recently been observed in GRB 051111, indicative of a high density medium in close proximity to a SSC or to the GRB (Berger et al. 2006; Prochaska et al. 2006). Berger et al. (2006) argue that the source of radiative pumping required for the excitation of the fine structure levels must be located close to the absorbing medium on the GRB line of sight. If this exciting source is a SSC, as they propose, the GRB is supposed to be located within the cluster - in contrast with our observations -, or there must be a chance alignment of the GRB and the SSC with its absorbing medium, which seems quite unlikely. Alternatively, Prochaska et al. (2006) argue that the absorbing medium could be circumstellar gas around the GRB progenitor star. Maintaining such a high density circumstellar envelope around a single star ejected with high velocity seems quite improbable. If such features turned out to be ubiquitous, our suggested scenario would probably require the ejection of a tight binary rather than that of a single star.

Clearly more detailed observations including accurate spatial information are needed to progress on these issues.

\section{Conclusion}

We have reported here the detailed properties of the environment of the four closest GRBs known to date, all of them being associated with SNe. For three of them (GRB 980425, GRB 020903, and GRB 031203) we have spectra of sufficient $\mathrm{S} / \mathrm{N}$ allowing us to examine the presence of WR stars, and all of them show the characteristic WR bump. HST images are also available for the GRB 980425, GRB 020903 and GRB 030329 hosts.

WR galaxies are thought to be related to very recent starbursts $(\leq 10 \mathrm{Myr})$. It is a very heterogeneous class of galaxies, occurring in various environments (Schaerer \& Vacca 1998). Following the Conti (1991) classification (more than 100 WR stars per WR galaxy) the GRB hosts studied here are WR galaxies. Smith (1991) estimated that $10 \%$ of HII galaxies are indeed WR galaxies. This somewhat supports the link between GRB and WR stars, i.e. GRBs with very recent formation of massive stars.

However, GRBs occurred at several hundreds of parsec away from the massive star forming regions hosting large numbers of $\mathrm{O}$ and WR stars. We notice that this applies to all the GRB hosts for which we have enough spatial resolution (see Fig. 4). Assuming this is purely a coincidence is not very plausible and would leave open the question of the formation of GRB/SNIbc in a medium containing only a small number of massive stars (e.g. less than $10 \mathrm{O}$ stars within a radius of $100 \mathrm{pc}$ around the SN1998bw region). We believe that such a coincidence is not fortuitous and propose therefore a new scenario, where GRBs are associated with runaway stars ejected from regions showing strong and recent star formation.

Our observations support the collapsar model by revealing for the first time the link between WR stars and GRBs associated with SNIbc, although in a less direct way than could have been expected. In our proposed scenario the frequency/rareness of the GRB events could be related to dynamical processes occurring during the cluster ejection. It is also very likely that additional parameters, such as metallicity and others suggested earlier, play a role in establishing the necessary conditions for a GRB event. The location of GRBs in sub-luminous galaxies and the absence of GRBs in massive galaxies (Le Floc'h et al. 2003, 2006; Fruchter et al. 1999), as well as the measurement of sub-solar metallicities in GRB hosts and nearby regions (e.g. Prochaska et al. 2004, and this paper), show that metallicity also plays a role. This question will be addressed in more detail in a subsequent paper. 
The discovery of a possible link between runaway WR massive stars and GRBs requires further confirmation. Massive binary scenarios must also be explored and tested. It has to be understood if such mechanisms occur preferentially in regions with a low density of stars or not. We hope that further nearby GRBs will be soon discovered by Swift and other satellites. Follow-up should include very high resolution images from the HST at several different epochs, and deep spectroscopy of the region hosting the GRB. X-shooter at VLT, equipped with an integral field unit will be ideal for the latter purpose.

Our results illustrate the need for a better understanding of the physics of massive stars and the importance of dynamical processes in cluster environments. It is still unclear whether the above results support the use of GRBs to test cosmological models.

Acknowledgements. The authors have benefited from interesting discussions with various colleagues including Francois Spite, Georges Meynet, Frederic Royer, Cristina Chiappini, Marc Freitag, and Willem-Jan de Wit. Gurvan Bazin contributed to a preliminary preparation of this work. We thank the referee for thoughtful and constructive comments and questions. Part of this work was supported by the Swiss National Science Foundation.

\section{References}

Allende Prieto, C., Lambert, D. L., \& Asplund, M. 2001, ApJ, 556, L63 Bally, J., \& Zinnecker, H. 2005, AJ, 129, 2281

Beck, S. C., Turner, J. L., Ho, P. T. P., Lacy, J. H., \& Kelly, D. M. 1996, ApJ, 457,610

Berger, E., Cowie, L. L., Kulkarni, S. R., et al. 2003, ApJ, 588, 99

Berger, E., Penprase, B. E., Fox, D. B., et al. 2005, ApJ, submitted [arXiv: astro-ph/0512280]

Blaauw, A. 1993, Massive Stars: Their Lives in the Interstellar Medium, ed. J. P. Cassinelli, \& E. B. Churchwell, ASP Conf. Ser., 35, 207

Bloom, J. S., Kulkarni, S. R., \& Djorgovski, S. G. 2002, AJ, 123, 1111

Caputi, K. I., Dunlop, J. S., McLure, R. J., \& Roche, N. D. 2005, MNRAS, 361, 607

Christensen, L., Hjorth, J., \& Gorosabel, J. 2004, A\&A, 425, 913

Conti, P. S. 1991, ApJ, 377, 115

de Wit, W.-J., Testi, L., Palla, F., \& Zinnecker, H. 2005, A\&A, 437, 247

Foley, R. J., Papenkova, M. S., Swift, B. J., et al. 2003, PASP, 115, 1220

Fruchter, A. S., Thorsett, S. E., Metzger, M. R., et al. 1999, ApJ, 519, L13

Fruchter, A., Levan, A., Hook, R., et al. 2003, GCN2243

Fryer, C. L., \& Woosley, S. E. 1999, ApJ, 526, 152

Fynbo, J. P. U., Holland, S., Andersen, M. I., et al. 2000, ApJ, 542, L89

Fynbo, J. P. U., Jakobsson, P., Møller, P., et al. 2003, A\&A, 406, L63

Fynbo, J. P. U., Sollerman, J., Hjorth, J., et al. 2004, ApJ, 609, 962

Galama, T. J., Vreeswijk, P. M., van Paradijs, J., et al. 1998, Nature, 395, 670

Galama, T. J., Vreeswijk, P. M., van Paradijs, J., et al. 1999, A\&AS, 138, 465

Gal-Yam, A., Moon, D.-S., Fox, D. B., et al. 2004, ApJ, 609, L59

Gies, D. R. 1987, ApJS, 64, 545

Guetta, D., Perna, R., Stella, L., \& Vietri, M. 2004, ApJ, 615, L73

Guseva, N. G., Izotov, Y. I., \& Thuan, T. X. 2000, ApJ, 531, 776

Hammer, F., Flores, H., Elbaz, D., et al. 2005, A\&A, 430, 115
Hirschi, R., Meynet, G., \& Maeder, A. 2005, A\&A, 443, 581

Hjorth, J., Sollerman, J., Møller, P., et al. 2003, Nature, 423, 847

Hobbs, G., Lorimer, D., Lyne, A., \& Kramer, M. 2005, MNRAS, 360, 974

Izotov, Y. I., \& Thuan, T. X. 1999, ApJ, 511, 639

Izotov, Y. I., Stasínska, G., Meynet, G., Guseva, N. G., \& Thuan, T. X. 2006, A\&A, in press [arXiv: astro-ph/0511644]

Izzard, R. G., Ramirez-Ruiz, E., \& Tout, C. E. 2004, MNRAS, 348, 1215

Jakobsson, P., Bjørnsson, G., Fynbo, J. P. U., et al. 2005, MNRAS, 362, 245

Klose, S., Greiner, J., Rau, A., et al. 2004, AJ, 128, 1942

Le Floc'h, E., Duc, P.-A., Mirabel, I. F., et al. 2003, A\&A, 400, 499

Le Floc'h, E., Charmandaris, V., Forrest, W. J., et al. 2006, ApJ, submitted

Leonard, P. J. T. 1991, AJ, 101, 562

Leonard, P. J. T. 1995, MNRAS, 277, 1080

Leonard, P. J. T., \& Duncan, M. J. 1990, AJ, 99, 608

Liang, Y. C., Yin, S. Y., Hammer, F., Deng, L. C., \& Flores, H. 2006, A\&A, submitted

MacFadyen, A. I., \& Woosley, S. E. 1999, ApJ, 524, 262

Malesani, D., Tagliaferri, G., Chincarini, G., et al. 2004, ApJ, 609, L5

Meynet, G., \& Maeder, A. 2002, A\&A, 381, 25

Meszaros, P. 2002, ARA\&A, 40, 137

Mirabal, N., Halpern, J. P., Chornock, R., et al. 2003, ApJ, 595, 935

O'Connell, R. W., Gallagher, J. S., III, \& Hunter, D. A. 1994, ApJ, 433, 65

Patat, F., Cappellaro, E., Danziger, J., et al. 2001, ApJ, 555, 900

Petrovic, J., Langer, N., Yoon, S.-C., \& Heger, A. 2005, A\&A, 435, 247

Podsiadlowski, Ph., Mazzali, P. A., Nomoto, K., Lazzati, D., \& Cappelaro, E. 2004, ApJ, 607, L17

Portegies Zwart, S. F., Makino, J., McMillan, S. L. W., \& Hut, P. 1999, A\&A, 348,117

Prochaska, J. X., Bloom, J. S., Chen, H.-W., et al. 2004, ApJ, 615, 625

Prochaska, J. X., Chen, H.-W., \& Bloom, J. S. 2006, ApJ, submitted [arXiv:astro-ph/0601057]

van Putten, M. H. P. M. 2004, ApJ, 611, L81

Schaerer, D., \& Vacca, W. D. 1998, ApJ, 497, 618

Schaefer, B. E., Gerardy, C. L., Höflich, P., et al. 2003, ApJ, 588, 387

Shaw, R. A., \& Dufour, R. J. 1994, Astronomical Data Analysis Software and Systems III, ed. D. R. Crabtree, R. J. Hanisch, \& J. Barnes, ASP Conf. Ser., 61,327

Smith, L. F. 1991, in Proceedings of the 148th Symposium of the IAU, held in Sydney, Australia, July 9-13, 1990, ed. R. Haynes, \& D. Milne (Dordrecht: Kluwer Academic Publishers), 267

Smith, R. C., Kirshner, R. P., Blair, W. P., Long, K. S., \& Winkler, P. F. 1993, ApJ, 407, 564

Soderberg, A. M., Kulkarni, S. R., Berger, E., et al. 2004a, ApJ, 606, 994

Soderberg, A. M., Kulkarni, S. R., Berger, E., et al. 2004b, Nature, 430, 648

Soderberg, A. M., Kulkarni, S. R., Fox, D. B., et al. 2005, ApJ, 627, 877

Sokolov, V. V., Fatkhullin, T. A., Castro-Tirado, A. J., et al. 2001, A\&A, 372, 438

Sollerman, J., Østlin, G., Fynbo, J. P. U., et al. 2005, NewA, 11, 103

Stanek, K. Z., Matheson, T., Garnavich, P. M., et al. 2003, ApJ, 591, L17

Starling, R. L. C., Wijers, R. A. M. J., Hughes, M. A., et al. 2003, MNRAS, 360, 305

Tenjes, P., Einasto, J., Maintzen, H. M., \& Zinnecker, H. 2005, A\&A, 369, 530

Turner, J. L., Beck, S. C., Crosthwaite, L. P., et al. 2003, Nature, 423, 621

Vanzi, L., \& Sauvage, M. 2004, A\&A, 415, 509

Woosley, S. E., Eastman, R. G., \& Schmidt, B. P. 1999, ApJ, 516, 788

Woosley, S. E., \& Heger, A. 2006, ApJ, ApJ, 637, 914

Yoon, S.-C., \& Langer, N. 2005, A\&A, 443, 643 